\title{
Psychoanalytisches «Verstehen» im Fall einer schizophrenen Patientin
}

\author{
Julia Braun (Zürich)
}

Zusammenfassung: Anliegen dieses Artikels ist es, zu zeigen, wie hilfreich der Ansatz von Josi Rom für die Entwicklung eines psychodynamischen Verständnisses schizophrener Menschen in der therapeutischen Arbeit ist. Die drei von Benedetti in «Todeslandschaften der Seele» dargelegten Primärsymptome der Schizophrenien, Spaltung, Autismus und Athymie, dienen als theoretische Aspekte seines Ansatzes. Die Autorin erläutert deren Bedeutung anhand eines Fallbeispiels aus ihrer psychotherapeutischen Praxis mit schizophrenen Patienten. Im Therapieverlaufwerden zwei verschiedene Therapiephasen aufgrund der unterschiedlichen Intensität des Kontaktwiderstandes unterschieden. Diesem kommt für die Behandlung von schizophrenen Patienten eine zentrale Rolle zu. Gegenstand der Auseinandersetzung wird auch die Reinszenierung der Psychose in der Teamdynamik sowie die Problematik des Therapieendes sein.

Schlüsselwörter: Schizophrenie, Spaltung, Fragmentierung, Autismus, Athymie, Kontaktwiderstand, Inhaltswiderstand

\section{Einleitung}

Anhand eines klinischen Beispiels zeige ich, stellvertretend für viele andere Therapieverläufe auf, wie Schizophrenien aus psychodynamischer Sicht modellhaft verstanden werden können.

Ich war mehrere Jahre lang als Psychotherapeutin in einer Spezialeinrichtung für an einer Psychose erkrankte junge Erwachsene tätig. Viele der Patienten hatten eine oder mehrere akut psychotische Phasen einer paranoiden Schizophrenie (ICD-10 F20.0) mit Positivsymptomatik (Wahnvorstellungen, Halluzinationen, Ich-Erlebnis-Störungen) hinter sich und litten nun überwiegend unter einer Negativsymptomatik (Antriebsmangel, Affektarmut, Aufmerksamkeitsstörungen, Alogie, Asozialität). Ihr Alltag wurde durch Dienste in den betreuten Wohnheimen, in denen sie lebten, Arbeitstherapie in verschiedenen handwerklichen Bereichen sowie unterschiedliche Freizeitangebote strukturiert. Zudem gab es verschiedenste therapeutische Angebote, wie eine Psychoedukationsgruppe, Physio-, Kunst- und 
Ergotherapie. Die Patienten erhielten eine psychiatrisch-psychotherapeutische Einzelbehandlung, wobei verschiedene Therapieschulen vertreten waren. Ich selber nahm eine psychodynamische Blickrichtung ein. Erreichten die Patienten die von der Institution vorgegebenen notwendigen Voraussetzungen, konnten sie sich im Rahmen eines Praktikums erproben und im besten Fall eine Ausbildung beginnen.

Bei der Patientin, auf deren Therapieverlauf ich nach einigen theoretischen Ausführungen ausführlicher zurückkommen werde, handelt es sich um eine Frau Anfang zwanzig, die gemeinsam mit zwei jüngeren Geschwistern bei ihren Eltern aufgewachsen war. Vater und Mutter waren verheiratet und befanden sich in stabilen Anstellungsverhältnissen. Die Patientin hatte einen mittleren Schulabschluss absolviert und eine Ausbildung begonnen, die sie aufgrund einer akut psychotischen Symptomatik im Rahmen einer paranoiden Schizophrenie abbrechen musste. Ihre Symptomatik beinhaltete Wahnwahrnehmungen, Gedankenabreissen sowie akustische Halluzinationen in Form einer Stimme, die meistens wohlwollend zu ihr sprach. Aufgrund einer massiven Gewichtsreduktion und Weinkrämpfen wurde sie in eine akutpsychiatrische Klinik eingewiesen, in der sie einige Monate lang klassisch psychiatrisch behandelt wurde. Eine weitere mehrmonatige Behandlung in einer Tagesklinik folgte und schliesslich der Aufenthalt in unserer Einrichtung.

In der ersten Zeit nach ihrem Eintritt in unsere Institution gab die Patientin kurzzeitig auftretende akustische Halluzinationen in Stresssituationen an. Sie berichtete mir im einzeltherapeutischen Setting, in dem ich sie einmal pro Woche sah, eindrücklich, wie sie ihre halluzinierten Stimmen selber aktiv beeinflussen konnte. Überwiegend litt sie jedoch unter einer ausgeprägten «Negativsymptomatik», die sie an der Fortsetzung ihrer Ausbildung hinderte und sie auch in der Bewältigung ihres Alltags deutlich beeinträchtigte. Es war schwierig, mit ihr in Kontakt zu kommen, sie sprach wenig und nahm auch zu Mitpatienten kaum Kontakt auf. Man konnte beobachten, wie sie sehr verlangsamt in gleichförmigen Bewegungen, abwesend wirkend, oft mit geschlossenen Augen einer einfachen Tätigkeit im Arbeitstherapiebereich nachging. Manchmal sass sie unbeweglich, müde wirkend über lange Zeiträume hinweg einfach da.

In der Institution geschah es wiederholt, dass insbesondere neue Mitarbeitende dazu tendierten, die schizophrenen Patienten für faul, unmotiviert oder widerständig zu halten. Josi Rom weist in seinem Buch «Identitätsgrenzen des Ich» auf diese Gefahr hin: «Solche Fehleinschätzungen haben Konsequenzen in unserem Fühlen dem Patienten gegenüber und können so auch therapeutische Erfolge gefährden. Wir entwickeln zum Beispiel Wut, wenn Patienten «nichts tun», 
obschon wir uns bemühen und Angebote machen. Wir beharren auf Regeln, die uns wichtig erscheinen. Unsere Legitimation heißt dann, daß der Schizophrene doch Struktur braucht, um nicht zu zerfallen» (Rom 2007: 45).

In meiner Intervisionsgruppe, in der ja das Verstehen der Patienten eine wesentliche Rolle spielt, wurde ich während meiner Fallvorstellungen gefragt, woran denn erkennbar sei, dass der jeweilige Patient an einer Schizophrenie leide. Auch diese Frage gibt einen Hinweis darauf, wie entscheidend die Verbindung einer Beschreibung des von aussen Sichtbaren mit der Bedeutung, die eine solche Erkrankung für einen Menschen in seinem Erleben haben kann, ist.

\section{Die Primärsymptome der Schizophrenie}

In ICD-10 und DSM-IV wird die Diagnose der Schizophrenie durch einen Kriterien-Katalog festgelegt (Möller et al. 2005: 149). Anhand einer vordergründigen Orientierung am Auftreten von Symptomen allein lässt sich ein ausreichendes Verständnis vom innerpsychischen Geschehen eines an Schizophrenie erkrankten Menschen jedoch nicht entwickeln. Eine gute Grundlage für ein psychodynamisches Verständnis von Schizophrenien bietet der psychopathologische Ansatz von Rom, der sich auf Benedetti bezieht. Gaetano Benedetti beschreibt in seinem Buch «Todeslandschaften der Seele» u. a. primäre Schizophreniesymptome, abgeleitet aus «einem intrapsychischen Geschehen, dessen Ursache ohne Umwelt nicht denkbar wäre, wobei eine besondere Verarbeitung dieser Umwelt in Zusammenhang mit der Person des Kranken steht» (Benedetti 2003: 16). Josi Rom vergleicht die Therapie mit einem an Schizophrenie erkrankten Menschen mit dem Weg durch eine Wüste, auf dem der Therapeut aufgrund seiner Wahrnehmung, seines Verständnisses und seiner Interpretation der jeweiligen Situation dem Patienten wie ein Kompass bei der Orientierung in dieser Umwelt helfen kann (vgl. Rom 2007: 27-28). Im Folgenden werde ich zeigen, in welcher Weise Roms modellhaftes Verstehen der Psychopathologie und der Psychodynamik der schizophrenen Psychosen dem Therapeuten eine Unterstützung sein kann, so dass es ihm möglich ist, diese Kompass-Funktion während der Therapie von Menschen mit schizophrener Psychose zu übernehmen.

Sowohl Benedetti als auch Rom argumentieren auf der Basis von Freuds topischen Theorien des psychischen Apparates. Beide nehmen Bezug auf die Systeme Unbewusst, Vorbewusst, Bewusst sowie auf die drei Instanzen Es, Ich, Über-Ich. Benedetti ist der Ansicht, «daß bei schizophrenen Auflösungsprozessen

die drei psychischen 〈Provinzen` Es, Ich und Überich nicht in gleichem Umfang involviert werden» (Benedetti 2003: 81). DerVerlust der innerpsychischen Struktur 
vollziehe sich vorwiegend zu Lasten des Ich. Die Schizophrenie kann aus dieser Perspektive also als eine Ichkrankheit verstanden werden. Um zu veranschaulichen, welche Auswirkungen beispielsweise eine Schizophrenie auf das Ich haben kann, entwickelt Rom auf Basis der beiden o.g. topischen Theorien und insbesondere auf Basis des Drei-Instanzen-Modells von Freud das Ich-Struktur-Modell.

\section{Abbildung $1 \quad$ Das Ich-Struktur-Modell (Josi Rom)}

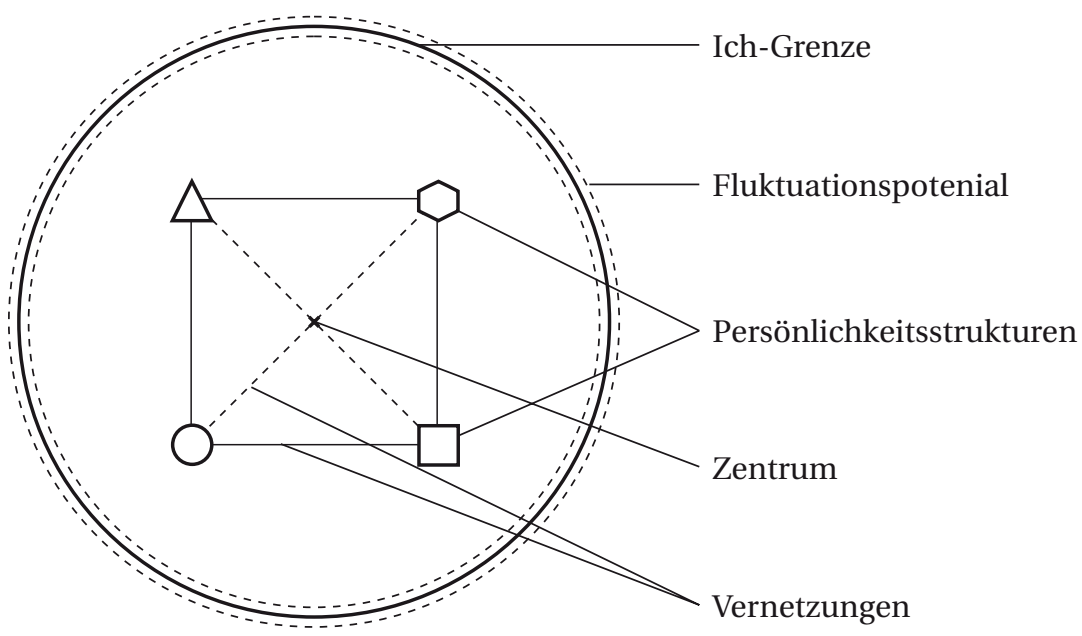

Sein Modell veranschaulicht Rom mit Hilfe obenstehender Abbildung (vgl. Rom 2007: 22). Es besteht aus Ich-Grenze, Persönlichkeitsstrukturen und Vernetzungen. Die Ich-Grenze ist dem Ich zugehörig und trennt es vom NichtIch. Beim gesunden oder neurotisch organisierten Ich wird die Ich-Grenze als ein stabiler Kreis dargestellt, ist als bildlicher Ausdruck für Funktionen zu denken, u. a. leicht durchlässig, kompakt und leicht elastisch, also nur wenig flexibel. Der Gesunde ist sich seiner selbst bewusst, kann sich abgrenzen und verfügt über eine funktionierende Realitätskontrolle. Die Persönlichkeitsstrukturen innerhalb des Ich einer Person unterscheiden sich im Detail und in ihren Funktionen voneinander. Sie passen sich beim gesunden Menschen der Bewegung des Gesamt-Ich an, ohne sich grundlegend zu verändern. Vernetzungen der Persönlichkeitsstrukturen untereinander und mit der Ich-Grenze ermöglichen Austausch und Koordination, 
das Funktionieren des Gesamt-Ich als Ich nach innen und nach aussen. Erst damit ist der Zusammenhalt des Ich-Bewusstseins gewährleistet (vgl. Rom 2007: 21-26).

Rom bezieht die von Benedetti beschriebenen Primärsymptome Spaltung (Fragmentierung) ${ }^{1}$, Autismus und Athymie auf sein Ich-Struktur-Modell und stellt die psychopathologischen Auswirkungen der Schizophrenie auf die Ich-Struktur zeichnerisch und damit bildlich vorstellbar dar. Nach Benedetti und Rom ist es dann sinnvoll von einer Schizophrenie zu sprechen, wenn sich die drei Primärsymptome bei der gleichen Person gleichzeitig oder in zeitlicher Nähe zeigen (vgl. Rom 2007: 27). In diesem psychoanalytischen Verständnis von Psychopathologie und Psychodynamik der Schizophrenien kann jede der je drei Erscheinungsformen der drei Primärsymptome auch als sekundäre, psychodynamische Folge der jeweils anderen Primärsymptome verstanden werden (vgl. Rom 2007: 48). Zur besseren Übersicht führe ich hier alle Primärsymptome mit ihren Erscheinungsformen tabellarisch auf:

\begin{tabular}{l|l|l|l}
\hline Primärsymptome & $\begin{array}{l}\text { Spaltung / } \\
\text { Fragmentierung } \\
\text { (Auflösung des Ich) }\end{array}$ & $\begin{array}{l}\text { Autismus } \\
\text { (Abkapselung des Ich) }\end{array}$ & $\begin{array}{l}\text { Athymie } \\
\text { (Energieverlust) }\end{array}$ \\
\hline Erscheinungsformen & $\begin{array}{l}\text { Gespaltene } \\
\text { Identitätsbildung, } \\
\text { Kohärenzverlust, } \\
\text { Ich-Entgrenzung }\end{array}$ & $\begin{array}{l}\text { Eigenweltlichkeit, } \\
\text { Ausdrucksunfähigkeit, } \\
\text { Selbst- und } \\
\text { Fremdverborgenheit }\end{array}$ & $\begin{array}{l}\text { Passivierung, } \\
\text { Devitalisierung, } \\
\text { Negativismus }\end{array}$ \\
\hline
\end{tabular}

Die Fragmentierung ist als Prozess vorstellbar, in dem sich das Ich auflöst. Dazu kommt es nach Rom dadurch, dass, bezogen auf das Drei-Instanzen-Modell von Freud, Es und Überich getrennt voneinander agieren. Sie haben unkoordiniert die Macht übernommen, so dass der Innendruck im Ich ständig zunimmt. «Die Ich-Grenze erstarrt dabei, verdichtet sich und wird spröde. Es ist der langfristig hoffnungslose Versuch, dem steigenden Innendruck etwas entgegenzusetzen, damit es nicht zur Fragmentation der Ich-Grenze und als Folge zum Verlust der Innenstrukturen und der Vernetzung des Gesamt-Ich, also zum schizophrenen Ich-Verlust oder zur Negativ-Existenz (...) kommt» (Rom 2007: 29). Demnach bewegen sich viele schizophrene Menschen auf einem Grad zwischen Stabilität und Ich-Verlust.

1 Rom zieht bezüglich des schizophrenen Geschehens den Begriff Fragmentierung dem der Spaltung vor, weil Spaltung oft mit Borderline-Störungen assoziiert werde, aber nach Rom nur wenig mit Spaltungsvorgängen in einem solchen Kontext zu tun habe (vgl. Rom 2007: 28). 


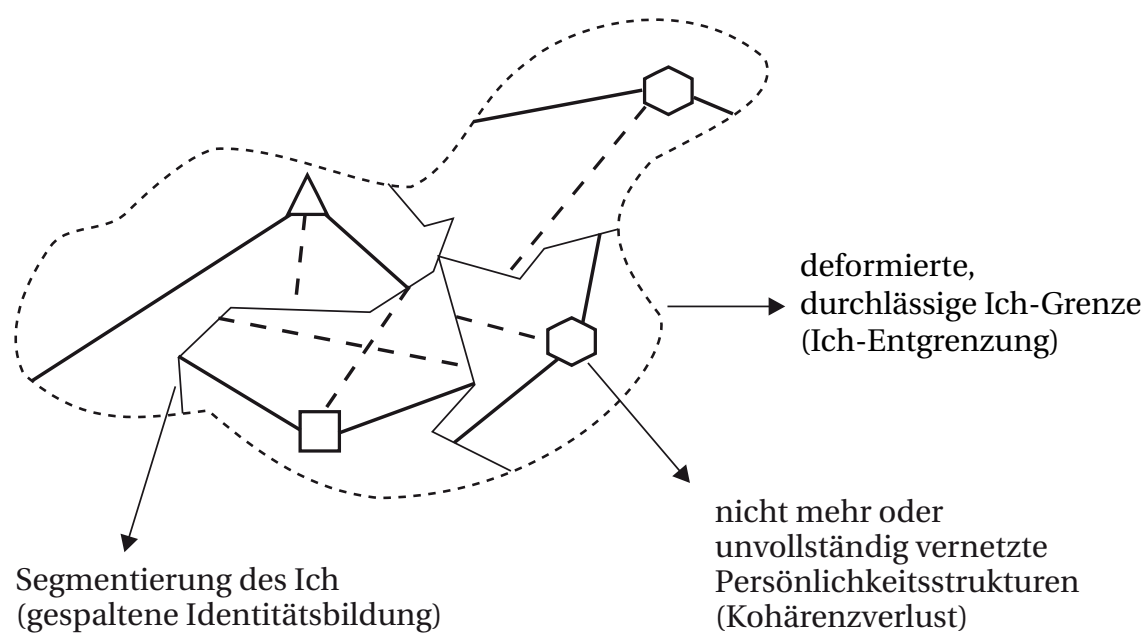

Gespaltene Identitätsbildung wird neben Kohärenzverlust und Ich-Entgrenzung als eine Erscheinungsform der Fragmentierung genannt. Das Ich ist in Roms Ich-Struktur-Modell segmentiert dargestellt. Rom beschreibt, dass die einzelnen dissoziierten Ich-Segmente weder gleichzeitig bewusst noch gleichzeitig emotional besetzt sind. Dies führt zwangsläufig zur Verkennung: Einzelne Ich-Segmente werden als Aspekte der Aussenwelt erlebt. In diesem Zusammenhang bezieht sich Rom auf Benedetti, der das Erleben der Fragmentierung anschaulich als ein Gefühl des schizophrenen Menschen beschreibt, in seinem Innern würden verschiedene Personen über ihn verhandeln. Dieses Gefühl werde mit der Gewissheit erlebt, diesen Teilpersonen völlig ausgeliefert zu sein, da ihnen kein Selbst gegenübergestellt werden könne (vgl. Rom 2007: 30). Der Kohärenzverlust wird nach Rom als Instabilität in der Vernetzung der Persönlichkeitsstrukturen untereinander beschrieben, die sich fragmentierend auf die Wahrnehmung und dadurch auch destabilisierend auf Selbst- und Fremdbild auswirkt (vgl. Rom 2007: 33). Laut Benedetti kann sich das als körperliches Gefühl einer inneren Unordnung zeigen. Der schizophrene Mensch erlebt seine Körperorgane als an andere Orte verschoben. In seinem Körper entstehen Löcher und Hügel oder eine andere Geschlechtszugehörigkeit, was sich bis zur Empfindung von körperlichem Zerfall steigern kann (Benedetti 2003: 18). So berichtete die Kunsttherapeutin unserer 
Institution gelegentlich sehr eindrücklich, auf welche Weise manche Patienten Figuren oder Köpfe plastizierten. Einzelne schizophrene Patienten modellierten bestimmte Körperteile an ungewöhnliche Stellen. Wenn die Kunsttherapeutin dies korrigierte, kam es wiederholt vor, dass das vorher korrigierte Körperteil beim nächsten Versuch vom Patienten an genau derselben «falschen» Stelle angebracht wurde. Beispielsweise wurden Augen auf die Stirn modelliert, was sich auch nach mehrfachen Hinweisen und Korrekturen der Kunsttherapeutin nicht verändern liess. Durch die Ich-Entgrenzung fallen nach Benedetti (vgl. 2002: 18) die Funktionen der Selbstabgrenzung und Objektbeziehung aus. Rom stellt dies in seinem Ich-Struktur-Modell in Form einer gestrichelten Linie als deformierte, durchlässige Ich-Grenze dar. Eine durchgehende, ungespaltene Ich-Grenze nach aussen fehlt. Das hat Auswirkungen auf das Gefühlsleben: Das Ich wird als von aussen einsehbar erlebt, so als sei der Zugriff von aussen stellenweise für andere freigegeben. Auch in die Gegenrichtung besteht das Gefühl, andere können das Ich mit beliebigen Inhalten von aussen durch die offenen Stellen anreichern. Rom übernimmt Benedettis Vorstellung, das Ich fliesse durch die fragmentierte IchGrenze aus und löse sich verschmelzend im Universum auf. So kann es sein, dass ein schizophrener Mensch gleichzeitig alles (Superexistenz) und nichts (Negativexistenz) ist (vgl. Rom 2007: 34-37). «Durch die passive Verschmelzung mit der Welt und dem Besetztwerden durch andere rettet sich das Ich vor dem völligen Verlust. Fragmentiert, autistisch abgekapselt und fremdbesetzt zu sein, ist besser als nicht zu sein. Autismus ist hier sowohl als aktive Abwehr als auch passives Primärsymptom zu verstehen» (Rom 2007: 35).

Nach Rom ist es leicht nachvollziehbar, dass es schwerste innere Desorientierung und Desorientierung in der Welt nach sich zieht, wenn ein Mensch gleichzeitig unter allen drei oben beschriebenen Formen der Fragmentierung leidet. Es ist gut vorstellbar, dass derjenige, der das erlebt, grosse Schwierigkeiten hat, sich zeitlich, örtlich und autopsychisch zu orientieren. Einfache Entscheidungsfragen können dann, wenn überhaupt, ambivalent bearbeitet werden. Und gerade diese Ambivalenz führt in eine Gefangenschaft, denn Entscheidungsfragen lassen sich nicht auflösen. Zur Auflösung wäre eine Entscheidung nötig, die ein schizophrener Mensch in einem solchen Zustand nicht treffen kann. Der Widerspruch wird festgehalten, und deshalb wird derWiderspruch zum Teil der eigenen Existenz. Es gibt also einen wesentlichen Unterschied zwischen der schizophrenen Ambivalenz und der gesunden oder neurotischen Ambivalenz. Wir sind ambivalent, weil wir uns nicht entscheiden können. Wenn sich ein Neurotiker entscheiden will, dann wägt er ab und sucht nach rationalen und gefühlsmässigen Kriterien. Eine 
Gewichtung der Argumente in einem breiten Kontext ist möglich. Die Frage nach der Wahl zwischen zwei verschiedenen Brotsorten kann durch eine mehr oder weniger eindeutige Entscheidung wieder aus dem Zentrum des Denkens eines neurotischen Menschen rücken. Einen schizophrenen Menschen besetzt die Frage vollständig. Die Argumente stehen sich gleichwertig gegenüber. Aufgrund der Athymie verfügt ein schizophrener Mensch nicht über die nötige Energie, um sich zu entscheiden. Die Folge ist Erschöpfung oder im schlimmsten Fall Katatonie. Als Lösungsmöglichkeiten stehen dem Schizophrenen autistisches Verschwinden aus der Beziehung, wahnhaftes Einbauen der Fragestellung und Entweichen durch einen Raptus zur Verfügung. Eine mögliche Abwehr des Schizophrenen ist die Rettung in ein eigenes Wahnsystem mit eigenen Gesetzen. Es können viele Jahre vergehen, bis ein einigermassen stabil-labiles Wahnsystem aufgebaut ist. Für den schizophrenen Menschen ist es ein Lebenswerk. Nach Rom darf Therapie keinesfalls darin bestehen, dieses Lebenswerk zu zerstören (vgl. Rom 2007: 35-37).

Abbildung 3 Autismus im Ich-Struktur-Modell (Josi Rom)

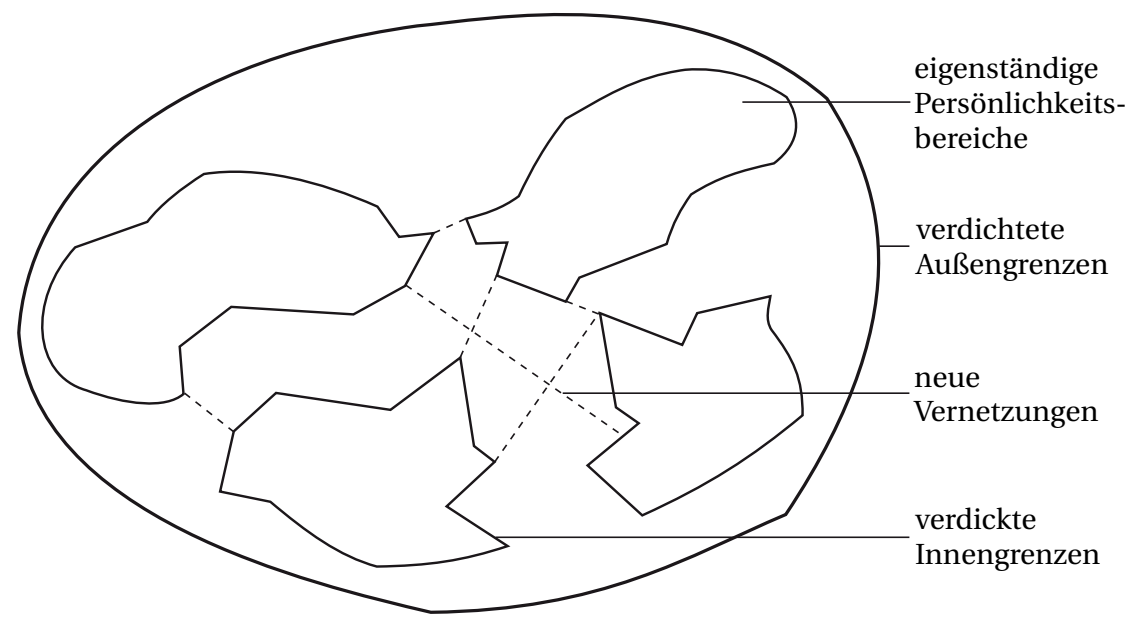

Psychosen - Psychoanalytische Perspektiven 
Der Autismus kann als Abkapselungsprozess des Ich verstanden werden. Er ist sowohl Primärsymptom als auch Abwehr und ist psychodynamisch eng mit der Fragmentierung verknüpft. Verglichen mit dem ursprünglichen Ich-StrukturModell kann man sich die Ich-Grenze im autistischen Zustand nach aussen sehr viel dichter und weniger durchlässig vorstellen. Das Ich funktioniert noch abgeschotteter von Es und Über-Ich. Auch innen sind die Grenzen verdickt. Die Persönlichkeitsbereiche sind eigenständig und neu vernetzt.

Eine Eigenweltlichkeit entsteht, die von aussen betrachtet sonderbar wirkt. Der Schizophrene spricht mit sich oder mit jemandem, der für andere nicht wahrnehmbar ist, sagt Unverständliches oder hat einen merkwürdigen Ausdruck in den Augen (Rom 2007: 38-39). So begegnete man auf dem Gelände unserer Einrichtung immer wieder Menschen, die irgendwo sassen, in der Gegend umherblickten, so als würden sie etwas mit den Augen fixieren und verfolgen, das für andere nicht sichtbar war. Manche sprachen vor sich hin oder antworteten auf Fragen, die für andere nicht hörbar gestellt waren. Die Ausdrucksunfähigkeit begründet Benedetti (2003: 25) damit, dass viele Erlebnisse des Schizophrenen in dessen eigenerWelt entstehen und oft ausserhalb von Sprache und Verständigung liegen. Dies mache es schwer, solche Erlebnisse für andere verständlich $\mathrm{zu}$ formulieren. Benedetti weist auch darauf hin, dass in der Psychopathologie ein zu hohes Gewicht auf die autistische Zurückgezogenheit gelegt werde. Der Kranke ringe jedoch darum, das nicht Sagbare ausdrücken zu können. Rom (2007: 40-41) sieht gerade die Ausdrucksunfähigkeit als eine therapeutische Herausforderung. Er weist auf die Gefahr hin, dass der Schizophrene im Autismus untergehe, wenn der Therapeut diese Herausforderung nicht annehme. Wichtig sei dabei, dass der Therapeut seine Grenze des Verstehens benenne. Es brauche viel Geduld, Zeit und ständiges, interessiertes und nichtbedrängendes Nachfragen, möglicherweise über viele Jahre hinweg. Die Selbst- und Fremdverborgenheit erklärt Rom damit, dass Worte und Bilder im Erleben des Kranken ständig zerfallen, so dass er sich selbst immer fremder wird. Schliesslich greifen seine psychotischen Mechanismen nicht mehr. Er ist verwirrt, sich selbst unweigerlich verborgen und somit auch für andere (vgl. Rom 2007: 42).

Die Athymie versteht Rom im Sinne von Huber (1961) am ehesten als Verlust des energetischen Potentials. Auch sie kann einerseits als Primärsymptom, andererseits als psychodynamische Folge von Fragmentierung oder Autismus verstanden werden.

Die Passivierung stellt sich nach Benedetti (2003: 28) im von aussen sichtbaren Verhalten des Patienten dar. Insbesondere in den Frühphasen der Psychose zieht er sich zurück und tut scheinbar überhaupt nichts mehr. Dies steht in starkem 
Kontrast zu dem, was der Patient selbst erlebt, nämlich einer hohen Aktivität, die er dazu aufwenden muss, er selbst zu bleiben. Diese Aktivität wird durch Passivität verdeckt, so dass sie von aussen kaum wahrnehmbar ist. Wenn wir uns tief auf unsere Gegenübertragungsgefühle einlassen und sie reflektieren, dann können sie nach Rom (2007: 44) ein Zugang zu der verdeckten Aktivität des Patienten sein. Laut Rom benötigt der Patient in der Passivität der Athymie «unser Mitgefühl und unser passives Dabeisein im Sinne der Entlastung, damit er im dualen Raum der Therapie loslassen kann und sich für das gegenwärtige Geschehen in seinem Erleben nicht mehr allein verantwortlich fühlen muss» (Rom 2007: 46). Devitalisierungbeschreibt ein Stillstehen der Zeit im Erleben des Patienten, das wir in der Gegenübertragung als etwas Zeitloses, Unendliches, das der Patient ausstrahlt, wahrnehmen können (vgl. Rom 2007: 46). In der Institution, in der ich arbeitete, lebten einige Patienten, die das Gefühl bei mir auslösten, sie lebten schon ewig dort - ein Gefühl von Unendlichkeit oder gar Zeitlosigkeit. Negativismus bezeichnet nach Benedetti (2003: 30) «den Widerstand des Kranken gegen alle Zuwendung seiner Mitmenschen und die Umkehrung ihrer Aufforderungen in ein gegenteiliges Verhalten.» Das negative Seinsgefühl und die Destruktivität des schizophrenen Menschen drücken sich im Negativismus auf der Handlungsebene aus. Eine affektive Grundlage fehlt sowohl für den Kontakt zu einem anderen Menschen als auch zum Treffen einer Entscheidung (vgl. Benedetti 2003: 30). Zum Negativismus als sekundäre, psychodynamische Folge der anderen Primärsymptome führt Rom aus, dass sowohl Fragmentierung (Auflösung des Ich) als auch Autismus (Abkapselung des Ich) von der Möglichkeit, mit einem anderen Menschen zu kommunizieren, wegführen. Das Ich verliert sich letztendlich, «entweder in der Auflösung im Nichts (Negativexistenz) respektive der Fusion mit der Welt (Superexistenz) oder in der Verdichtung im Eigenen, Eigenweltlichen mit der Folge der Selbst- aber auch Fremdverborgenheit» (Rom 2007: 47-48). Im Negativismus finde der Schizophrene einen Weg, sich dialogisch abzugrenzen. Er beweise sich seine Existenz, indem er sich der Zuwendung seines Mitmenschen entgegenstelle. Das Entgegenstellen als Beweis der eigenen Existenz sei so entscheidend, dass der schizophrene Mensch dafür sogar bereit sei, auf eben diese Zuwendung seines Gegenübers zu verzichten. Würde er die Zuwendung annehmen, würde sich der Schizophrene durch Fusion mit dem Anbieter der Zuwendung verlieren, was eine Weiterführung der Fragmentation bedeuten würde. Durch diesen enormen Verzicht wird deutlich, mit welcher Verzweiflung der Schizophrene versucht, sich zu retten. Rom beschreibt den Negativismus als verzweifelte Leistung der Selbstrettung, als Antwort auf den Ich-Verlust (Rom 2007: 47-48).

Psychosen - Psychoanalytische Perspektiven 


\section{Fallbeispiel}

Erste Therapiephase

Die o.g. Patientin wies also nicht nur einen Grossteil der im ICD-10 beschriebenen Symptomatik auf, sondern sie zeigte auch die von Rom und Benedetti beschriebenen Primärsymptome. So war sie beispielsweise im Sinne der Ich-Entgrenzung der Ansicht, jeder kenne die Gedanken der anderen und beschwerte sich in einer Therapiesitzung verärgert darüber, dass sie nicht verstehe, warum die Leute nicht sagen, was sie denken, wo doch sowieso klar sei, was sie denken würden, sie also keinen Grund hätten, es nicht auszusprechen. Von aussen betrachtet bekam man schnell den Eindruck, die Patientin lebe in ihrer eigenen Welt, sie schien häufig auf der sprachlichen aber auch auf derVerhaltensebene für andere unzugänglich. Bei dem was sie tat, wirkte sie sehr passiv. Oft schien es, als tue sie nichts. Wenn sie eine Aufgabe bekam, dann führte sie diese in der Regel anders aus als vorgegeben. Beispielsweise kürzte sie ein Werkstück um 18 statt um $15 \mathrm{~cm}$. Häufig musste sie eigene Arbeiten wegwerfen, da sie nicht mehr verwendbar waren, was ihr nichts auszumachen schien. Möglicherweise konnte es ihr nur auf diese Weise gelingen, sich im Sinne des Negativismus dialogisch abzugrenzen und damit vor der Fusion mit dem Arbeitstherapeuten, d. h. einer weiteren mit IchVerlust verbundenen Fragmentierung, zu schützen. An dieser Stelle wird besonders deutlich, wie entscheidend eine psychodynamische Sichtweise auf die Patienten sein kann. Fehlt diese, kann sich das im Umgang mit ihnen negativ auswirken. Aus einem Alltagsverständnis heraus beispielsweise könnte man viele der schizophrenen Patienten für zu wenig intelligent oder zu faul halten. Meiner Erfahrung nach entsteht ein solcher Eindruck im täglichen Umgang mit schizophrenen Patienten trotz des Wissens um das bestehende Krankheitsbild schnell.

Ausgehend von den Aspekten der Passivierung und Devitalisierung werden die Schwierigkeiten verständlicher, die mir in der ersten Therapiephase beim Versuch, mit der o.g. Patientin in Kontakt zu kommen, begegneten. Sie äusserte sich wenig. Wenn sie sich äusserte, dann meistens sehr knapp in Form eines Achselzuckens oder eines kurzen Satzes. Ich war in den therapeutischen Gesprächen darauf bedacht, keine allzu langen, mit Schweigen gefüllte Zeiträume entstehen zu lassen. Dies erforderte eine hohe Aktivität meinerseits. Gleichzeitig war ich bemüht, möglichst entspannt zu sein, meine Gegenübertragungsgefühle wahrzunehmen und zu verstehen, was gerade zwischen der Patientin und mir passiert. Als ich einmal in die Werkstatt kam, um mit dem Arbeitstherapeuten etwas zu besprechen, begegnete ich dort meiner Patientin. Sie sass, wie ich es schon öfter gesehen hatte, seitlich an ihrer Werkbank und blickte abwesend wir- 
kend vor sich hin. Da ich auf den Arbeitstherapeuten warten wollte, fragte ich sie, ob ich mich zu ihr setzen dürfe. Sie nickte. So sassen wir einige Minuten lang schweigend nebeneinander, wobei ich ein gutes Gefühl hatte. In diesem Moment, ausserhalb des therapeutischen Settings, war ich besonders entspannt, frei von Ansprüchen oder Erwartungen. Als ich schliesslich aufstand, verabschiedete sich die Patientin von mir und wünschte mir ein schönes Wochenende. Sie blickte mich mit präsenter Offenheit an und ich hatte ein Gefühl von Kontakt, das ich in den vorangegangenen Therapiestunden immer wieder vermisst hatte. Vorstellbar ist, dass die Patientin aufgrund meines passiven Dabeiseins entlastet war. Die Bedeutung von Gelassenheit für eine Therapie mit schizophrenen Patienten wurde mir durch diese Begegnung deutlicher und ich überlegte, dass es schön wäre, es könnte mir gelingen, diese mit in die therapeutischen Gespräche zu nehmen. Die Erinnerung an diese Begegnung mit der Patientin half mir in den folgenden Therapiestunden, mich entspannter zurückzulehnen.

\section{Kontaktwiderstand in der ersten Therapiephase}

Da ich überwiegend mit schizophrenen Patienten arbeitete, begegneten mir häufig Schwierigkeiten in der Therapie, die sich u. a. aus der oben beschriebenen Symptomatik erklären. Dies waren Phänomene wie Verspätungen, das NichtWahrnehmen von vereinbarten Terminen, langem Schweigen oder sehr knappen Antworten im Gespräch sowie ein vorzeitiges Beenden der Stunde. So auch bei meiner schon erwähnten Patientin. Insgesamt war sie sehr wortkarg. Ihre Mimik veränderte sich kaum. Sie bewegte sich selten und schien möglichst wenig von sich zeigen zu wollen.

Rom erwähnte auch in seinen Seminaren immer wieder den Kontaktwiderstand, eine Form des Widerstandes bei schizophrenen Patienten, dem Benedetti (1975) in seinen «Ausgewählten Aufsätzen zur Schizophrenielehre» ein ganzes Kapitel widmet. Benedetti unterscheidet zwei strukturell verschiedene Widerstände. Neurotische Patienten seien grundsätzlich mit dem Kontakt zum Therapeuten einverstanden und bereit, von ihren Sorgen und Problemen zu berichten, in der Hoffnung, Unterstützung zu bekommen. Sie entscheiden sich freiwillig dazu. Widerstand zeigt sich bei ihnen infolge einer Konfrontation durch den Therapeuten mit inhaltlich für sie unangenehmen Themen, denen sie sich verschliessen möchten. Bei neurotischen Patienten handelt es sich demnach meistens um einen Inhaltswiderstand. Dieser entwickelt sich auch bei schizophrenen Patienten. Sie haben aber häufig solche «Angst davor, mit einem fremden Menschen, dem Therapeuten, in einen seelischen Kontakt zu treten, ihm eine Seite» (Benedetti 
1975: 145) ihres Selbst anzuvertrauen, dass eine Arbeit an für den Patienten unangenehmen Inhalten zunächst versperrt ist. Benedetti bezeichnet diese besondere Art des Widerstandes, den wir bei neurotischen Patienten so regelmässig nicht finden, als Kontaktwiderstand. Dieser sei meist dem Inhaltswiderstand sozusagen vorgelagert, weshalb er in der Behandlung eines psychotischen Patienten immer zuerst bearbeitet werden müsse. Dies stelle eine wesentliche Voraussetzung dar, um sich nachfolgend dem Inhaltswiderstand widmen zu können.

Bei meiner schizophrenen Patientin merkte ich, dass ein Kontakt nur schwer herstellbar war. Oft fielen ihr die Augen zu. Auch sprachlich war sie selten erreichbar. Es gab wenige Augenblicke, in denen sie unerwartet schnell mehrere Sätze hintereinander sprach. In solchen seltenen Momenten war ich erstaunt darüber, wie viel sie von dem realen äusseren Geschehen um sie herum erfasste, da sie im Allgemeinen recht abwesend und müde wirkte. Nach einigen Stunden entdeckte ich ein Thema, das sie zu interessieren schien. Es handelte sich um ihren Wunsch, möglichst bald eine Ausbildung zu beginnen. Sie äusserte ihn meist in Verbindung mit Andeutungen über bereits erfolgte Handlungen ihrerseits, wie das Einholen von Erkundigungen an Schulen oder das Abschicken von Bewerbungen. Ab und zu gelang eine Kommunikation darüber, aber auch die erlosch nach wenigen Minuten wieder. Wir hatten schon etliche Therapiestunden hinter uns und mein Gefühl von «nicht wirklich weiterkommen» verstärkte sich. Immer wieder fragte ich mich, wie ich einen Zugang zu dieser Patientin bekommen könnte.

Benedetti (1975: 145) empfiehlt, den Kontaktwiderstand früh zu deuten und korrektiv zu beantworten. Dabei meint er mit Deutung nicht die in der Neurosentherapie übliche Deutung des Inhaltswiderstandes, indem dem neurotischen Patienten sein Abwehrmechanismus aufgezeigt wird. Dies würde sich verstärkend auf den Kontaktwiderstand eines psychotischen Patienten auswirken, weil er sich abgelehnt fühlen würde. Wichtig sei es, «von Anfang an auf die Angst des Patienten in der Kontaktnahme hinzuweisen, damit sich dieser von seinem Therapeuten verstanden fühlt» (Benedetti 1975: 145). Besonders dann, wenn der Patient nicht antworte, könne das in Form eines therapeutischen Monologes stattfinden, in dem mögliche Gründe genannt werden, die Vertrauen erschweren und Schutz nötig machen. Zusätzlich soll der Kontaktwiderstand nach Benedetti korrektiv beantwortet werden, indem der Therapeut durch sein Verhalten diese Art des Widerstandes hinfällig werden lässt. Der Therapeut solle, so Benedetti, die Ängste und Unsicherheiten des Patienten respektieren und ihm keine belastende Kontakterfahrung vermitteln, indem er ihn drängt, alles zu sagen, was ihm in den Sinn kommt. 
Dies setzte ich in den Therapiestunden um, bemüht, nicht zu lange Schweigephasen entstehen zu lassen und im Hintergrund gleichschwebend aufmerksam zu sein. Es erforderte eine gewisse Anstrengung, die von mir als lang empfundene Zeit auszuhalten, in der kaum Bewegungen in der Therapie spürbar waren. Benedetti führt dazu ermutigend an: «Wenn man auch für die erste Phase der Therapie scheinbar viel Zeit braucht, indem man noch darauf verzichtet, Deutungen zu geben, welche die tiefsten Probleme des Patienten anfassen und Angst auslösen können, gewinnt man dafür Zeit in der zweiten Hälfte der Therapie, wenn das schwache Ich des Kranken stärker geworden ist» (Benedetti 1975: 146).

\section{Psychotische Dynamik im Team als Reinszenierung der Psychose der Patientin}

Wie erwähnt, hatte meine Patientin, wie viele andere in der Institution lebende Patienten auch, aufgrund ihrer Erkrankung eine begonnene Ausbildung abbrechen müssen und nun die Möglichkeit, nach erfolgreicher Therapie auf den verschiedenen Ebenen (psychiatrisch-psychotherapeutisch, sozial- und arbeitstherapeutisch), mit Unterstützung eine andere Ausbildung zu absolvieren. Bei uns angekommen wirkte sie überwiegend fremdmotiviert. Eltern und Klinik hatten ihr unsere Einrichtung empfohlen. Die Patientin war freundlich, zeigte sonst aber kaum Emotionen und nahm selten Kontakt zu anderen auf. Anfänglich wurde auch von Mitarbeitenden in der Institution berichtet, sie habe sich während ihres Aufenthaltes für verschiedene Ausbildungen ausserhalb der Institution beworben. Aus den verschiedensten Gründen sei es nie zu einer Realisierung des jeweiligen Ausbildungswunsches gekommen. Die starke Ambivalenz der Patientin wurde im therapeutischen Zweiergespräch u. a. in ihrer Frage an mich, «Denken Sie, ich bin in der Lage eine ... -ausbildung zu machen?», deutlich. Es zog sie weg aus der Institution und gleichzeitig schien sie selbst zu ahnen, in ihrem momentanen Zustand nicht in der Lage zu sein, selbständig zu leben und eine Ausbildung erfolgreich zu absolvieren. Insgesamt entwickelte sie sich im Vergleich zu den anderen Patienten sehr langsam, während die durch die Institution vorgegebenen Anforderungen stiegen. Beispielsweise war es für Mitarbeitende schwierig, mit ihr in Kontakt zu kommen, sie nahm wohlgemeinte Ratschläge nicht an und führte Aufträge nicht den Vorgaben entsprechend, sondern auf ihre eigene Weise aus. Oft sass sie über lange Zeiträume hinweg unbeweglich in der Werkstatt vor ihrer Arbeitsaufgabe. Sie löste Wut bei verschiedenen Mitarbeitern aus, die in Teamsitzungen berichteten, die Patientin würde nicht mitmachen und «sich sperren». Es kam zu Fehleinschätzungen, die ich immer wieder bemüht war aufzuklären. 
Da die Patientin wenig kommunizierte, gelang es auch mir oft nur auf einer eher allgemeinen als auf einer individuellen Ebene, Verständnis für sie zu erzielen.

Während einer Teambesprechung wurde ich von dem für die Patientin zuständigen Bezugsmitarbeiter (Sozialpädagoge) darüber informiert, dass sowohl ihre Mutter als auch ihr gesetzlicher Betreuer gerne wissen würden, wie es mit ihr weitergehe und deshalb zum Gespräch in die Einrichtung kämen. Er fragte mich, ob es nicht sinnvoll wäre, wenn auch ich bei diesem Standortgespräch anwesend wäre. Ein Termin war bereits vereinbart und die Beteiligten eingeladen. Ich bekam das Gefühl, die Patientin schützen zu wollen respektive sie dieser Situation mit den vielen Menschen nicht allein ausgesetzt sein zu lassen und sagte zu, sofern die Patientin selbst auch teilnehmen würde. Ich war bereit, das therapeutische Setting des Zweiergesprächs zu verlassen, um gemeinsam mit der Patientin diese Situation zu bewältigen. Ich vereinbarte also einen Termin mit der Patientin, um diese Frage mit ihr zu besprechen. Die Patientin wollte teilnehmen. Auch sie wollte wissen, wie es weitergehen könnte und war auch mit meiner Teilnahme an dem Gespräch einverstanden. In einem kurzen Vorgespräch mit dem Sozialpädagogen sagte ich, dass es mir wichtig sei, dass wir nichts über den Kopf der Patientin hinweg entscheiden. Als Gesprächsziel legten wir ein Aufzeigen verschiedener Perspektiven fest, die von dem Sozialpädagogen bereits vorbereitet waren. Er sagte mir, dass, da er selbst bald die Institution verlasse, der neue Bezugsmitarbeiter, der gerade neu eingestellt worden war, ebenfalls an dem Gespräch teilnehmen werde. Kurz vor Gesprächsbeginn bat mich der Sozialpädagoge, die Moderation des Gesprächs zu übernehmen, was mich überraschte. Ich entschied mich auch hier zuzusagen, da ich in der Übernahme der Moderation eine Chance sah, innerhalb der sich überschlagenden Ereignisse und der sich zu verselbständigen drohenden Dynamik eine gewisse Kontrolle über die Situation zu haben und damit eine Möglichkeit, die Patientin zu unterstützen.

Als ich den Gesprächsraum betrat, waren der aktuelle und der zukünftige Bezugsmitarbeiter, der gesetzliche Betreuer, die Patientin und ihre Mutter bereits um den Tisch versammelt. An der Stirnseite war ein Stuhl freigelassen worden, auf dem ich, nachdem ich alle einzeln begrüsst hatte, Platz nahm. Zunächst fragte ich die beiden von ausserhalb Angereisten nach ihren Anliegen. Der gesetzliche Betreuer ergriff zuerst das Wort. Er sagte, er habe mitbekommen, dass die Patientin vom Wohnheim eine Abmahnung bekommen habe und nun befürchte er, dass ihr Aufenthalt gefährdet sei. Weiter habe er den Eindruck, dass die Medikamente nicht richtig eingestellt seien, und frage sich, ob es nicht besser wäre, ein Medikament zu finden, das besser wirke. Die Mutter äusserte auf meine Frage nach ihrem Anliegen, 
dass sie sich Sorgen um ihre Tochter mache, da sie den Eindruck habe, es sei ihr schon besser gegangen.

Im Gespräch wurde über mögliche Zukunftsperspektiven für die Patientin diskutiert. Die Beteiligten gerieten erstaunlich schnell und wiederholt in einen Dialog über die anwesende Patientin, so dass ich überwiegend damit beschäftigt war, sie daran zu erinnern, dass die Person, um deren Zukunft und Befinden es im Moment gehe, anwesend sei, und es doch sinnvoll wäre, sie selbst zu fragen. Das wurde dann auch getan. Die Antworten der Patientin fielen sehr knapp aus und wurden oft missverstanden. Schnell gerieten die Gesprächsteilnehmer wieder in die Situation, über die anwesende Patientin zu reden, worauf ich immer wieder hinwies. Letztendlich waren verschiedene Möglichkeiten genannt worden, welche die Patientin ergreifen könnte, womit die beiden von ausserhalb angereisten Personen ganz zufrieden wirkten.

Anhand dieser Beschreibung wird deutlich, wie schnell sich die Psychose einer Patientin in Form einer psychotischen Dynamik auch in einem professionellen Team konkretistisch re-inszenieren kann. Benedetti führt zur Passivierung, einer der o. g. Erscheinungsformen der Athymie, aus: «Das Erleben der «Passivität» bedeutet, dass der Kranke zu einer Zielscheibe der Gebärden, Gedanken und Intentionen anderer Menschen geworden ist, die ihn nach Belieben formen und lenken» (Benedetti 2003: 28). Nach Benedetti erlebe sich der Schizophrene als Schauplatz von verschiedenen Kräften, die in seinem Innern um die Oberhand kämpfen. Dies sei dann ein Gefühl, als schaue er Ereignissen, die ihn eigentlich zentral angehen, unbeteiligt zu, so als würde ein Film vor ihm ablaufen (Benedetti 2003: 29). Es ist gut möglich, dass diese Beschreibung auch auf das Erleben meiner Patientin in ihrer Psychose zutrifft. Von dieser Annahme aus betrachtet hat sich ihr Psychoseerleben in dem Standortgespräch mit Menschen, die aus ihrer jeweiligen Funktion oder Beziehung zur Patientin heraus je eigene - durchaus gut gemeinte - Erwartungen an sie hatten, in der Realität re-inszeniert. Die im Anschluss an dieses Gespräch erfolgte Wende in der Therapie, auf die ich im nächsten Abschnitt eingehen werde, wäre vor diesem Hintergrund verständlicher. Die Patientin musste aufgrund ihres oben beschriebenen Zustandes, der u. a. geprägt war von Ausdrucksunfähigkeit, Devitalisierung und Selbst- und Fremdverborgenheit, grösstenteils wie eine Zuschauerin dabeisitzen. Anzunehmen ist, dass sie sich insbesondere aufgrund der Ich-Entgrenzung kaum schützen konnte, sogar eher im Sinne des Negativismus dazu neigte, gut gemeinten Ratschlägen zu widersprechen. Auf die meisten Beteiligten mochte das wiederum unbeteiligt und uneinsichtig wirken. 


\section{Zweite Therapiephase}

Erstaunt war ich, als die sonst so zurückhaltende Patientin sich beim Verabschieden am Ende des Standortgesprächs mit Nachdruck bei mir dafür bedankte, dass ich dabei gewesen sei, eine Geste, die ich im Nachhinein als Auftakt für eine zweite Therapiephase betrachte. In der darauffolgenden Therapiestunde erlebte ich die Patientin wie ausgewechselt. Sie berichtete spontan von Verschiedenem, was ihr durch den Kopf ging und bot mir eine Reihe von Themen an. Ich hatte erstmalig das Gefühl, dass ein wirklicher Dialog zwischen uns in Gang gekommen war. Auf meine Nachfrage hin konnte sie mir auch mitteilen, dass sie sehr froh darüber war, dass ich in der Gesprächsrunde den anderen Beteiligten nichts verraten hätte, was nicht zwischen uns vereinbart gewesen sei.

Das Verlassen des Settings des therapeutischen Zweiergesprächs hatte der Patientin ermöglicht, zu überprüfen, wie ich sie vor anderen Menschen vertrete. Offenbar hatte die Erfahrung, die sie in dem Standortgespräch mit mir gemacht hatte, eine angstreduzierende Wirkung, die sich vermindernd auf ihren Kontaktwiderstand auswirkte. Eine Entlastung trat ein, die bewirkte, dass die Patientin sich im dualen Raum der Therapie (angst)freier bewegen konnte. Zunächst wurde mir das an ihrer plötzlichen Verhaltensänderung deutlich. Ich bemerkte beispielsweise, dass sie, im Gegensatz zu vorher, den Therapieraum, in dem auch die bisherigen therapeutischen Sitzungen stattgefunden hatten, wahrzunehmen schien. Sie machte Bemerkungen über Gegenstände im Raum, redete insgesamt mehr und spontaner und sprach auch Belastendes an. Antwortlatenzen traten so gut wie nicht mehr auf. In der Folge konnte sie mir auf einer inhaltlichen Ebene viele Themen anvertrauen, die sie beschäftigten und ihr Sorge bereiteten.

Wie oben in den Ausführungen zur Passivität beschrieben, kann das, was wir in der Gesprächsrunde von aussen wahrgenommen haben, in grossem Kontrast zu dem gestanden haben, was die Patientin selbst erlebt hat. Da, wo sie als passiv wahrgenommen wurde, war sie möglicherweise sehr aktiv damit beschäftigt, sie selbst zu bleiben. Ich vermute, dass die Patientin durch meine Anwesenheit beim Standortgespräch und mein ständig auf ihre Anwesenheit verweisendes Verhalten das Gefühl hatte, dem Geschehen nicht allein ausgesetzt zu sein. Mein Verhalten insgesamt entsprach offenbar einer korrektiven Beantwortung des Kontaktwiderstandes, wie sie von Benedetti empfohlen wird. Indem ich der Patientin handelnd zeigte, dass ich ihre Ängste und Unsicherheiten respektierte und ihr keine zusätzliche belastende Kontakterfahrung vermittelte, war ihr Kontaktwiderstand nicht mehr in dem Ausmass notwendig wie zuvor. 
Sie hatte sich, wie sie deutlich zum Ausdruck brachte, gut durch mich vertreten gefühlt, so dass nun auch in den therapeutischen Zweiergesprächen ein Dialog zwischen uns in Gang kommen konnte. Nach weiteren Therapiestunden konnte sie mir mitteilen, dass sie Vertrauen zu mir habe und sich von mir verstanden fühle. Es gelang ihr, mir mitzuteilen, dass es ihrer Meinung nach aussichtslos sei, mit dem Betreuer oder ihrer Mutter über die Medikamente zu reden. Häufig fühlte sie sich von anderen missverstanden. Ein Aneinandervorbeireden erlebte sie als Phänomen, das ihr mit verschiedenen Menschen passierte und sie belastete. Die Frage, wie sie von anderen Menschen besser verstanden werden könne, trat in den Vordergrund. Ein weiteres Thema war die Scham darüber, dass sie erst dann eine Ausbildung beginnen, wenn ihre gleichaltrigen Freunde ihre Ausbildungen bereits erfolgreich abgeschlossen haben würden.

Auch in dieser zweiten Therapiephase geschah es wieder, dass der Patientin die Augen zufielen. Ich fragte sie, wie sich das anfühle, wenn ihr die Augen zufielen. Inzwischen hatte die Patientin genügend Vertrauen zu mir, um mir zu beschreiben, wie sie diesen Zustand erlebe. Sie beschrieb eindrücklich, wie man zuerst einen Gedanken habe und dann plötzlich weg sei. Weiter wies sie darauf hin, dass sie dieses Phänomen des «Weg-Seins» bereits aus der Schulzeit kenne. Sie habe sich immer weit nach hinten gesetzt, damit das nicht so auffalle. Ich bemerkte anerkennend, was für eine grosse Leistung es von ihr gewesen sei, mit diesem immer wieder «Weg-Sein» einen mittleren Schulabschluss erfolgreich zu absolvieren. Benedetti nennt dieses Phänomen die schizophrene negative Existenz. Er beschreibt die Erfahrung der Nicht-Existenz als eine Folge der Fragmentierung. Die psychischen Strukturen, denen wir das Bewusstsein verdanken, lösten sich auf. Die negative Existenz stelle die Erlebensseite dieses Ich-Zerfalls dar (Benedetti 2003: 48-49).

\section{Die Bedeutung des Abschieds in der Therapie mit schizophrenen Patienten}

«Das Zur-Sprache-Bringen des Therapieabschlusses sollte sich dem Entwicklungsstand der Behandlung und dem Patienten anpassen und ihn weder bedrohen noch überfordern» (Rom 2007: 223). Dementsprechend teilte ich meinen Einzeltherapiepatienten so früh, wie es mir möglich war, in einem extra hierfür anberaumten Gruppengespräch meinen anstehenden Weggang aus der Institution mit. Auch die oben genannte Patientin war bei diesem Gespräch anwesend. Direkte Reaktionen von ihr waren nicht sichtbar. Sie schaute auf ihre nervös spielenden Hände, während die anderen sich verbal dem Thema widmeten, indem sie nachfragten und Trauer oder Ärger äusserten. Als ich meine oben beschriebene Patientin im nächsten Einzelgespräch erneut auf unseren bevorstehenden 
Abschied ansprach, konnte sie sagen, dass sie das traurig fände. Uns standen zu diesem Zeitpunkt, kurz nachdem sich meine Patientin in der Therapie öffnen konnte und mir einen grossen Geschenkkorb voller Themen mitgebracht hatte, nur noch wenige Monate für die Therapie zur Verfügung. Dies stellte natürlich eine Überforderung für uns dar, da es nicht mehr möglich war, all diese Themen noch vor meinem Abschied zu besprechen. Ich teilte der Patientin mit, dass ich denke, dass diese Themen sehr viel Zeit füllen können und dass wir ja nur noch eine begrenzte Zeit zurVerfügung hätten, es also nicht schaffen würden, alle angesprochenen Themen zu bearbeiten und bat sie, ein Thema auszuwählen, das ihr besonders wichtig ist.

In Anbetracht des Therapieendes wurde eine erneute Zunahme des Kontaktwiderstandes deutlich, wobei dessen Intensität nicht soweit anstieg wie in der oben beschriebenen ersten Therapiephase. Beispielsweise entstanden erneut kürzere Schweigephasen. Diesmal bestand jedoch die Möglichkeit, auf die positiven Erfahrungen in der Therapie zurückzugreifen.

«Sobald sich das Ende einer Behandlung am Horizont zeigt, bewährt es sich, gemeinsam konkrete Phantasien dazu zu entwickeln. Von dieser Phantasie ausgehend, nähert man sich behutsam, aber stetig der Umsetzung in die Realität. Korrekturen der Annäherung der Phantasien an die Realität sind angebracht, soweit sie nicht im Dienst des Widerstandes stehen und die Therapie künstlich verlängern» (Rom 2007: 222). Eine von meiner Patientin geäusserte Phantasie war, wie auch zu Therapiebeginn geäussert, möglichst bald eine Ausbildung zu absolvieren, wozu sie jedoch aufgrund ihres damaligen gesundheitlichen Zustandes noch nicht in der Lage gewesen wäre. In einem solchen Fall ist es wichtig, einen Bezug zur Realität herzustellen, ohne den Wunsch der Patientin völlig zunichte zu machen. Rom empfahl mir in der Supervision, der Patientin etwas mitzuteilen wie: «Ich sehe, dass sie weiter an Ihrem Traum festhalten, irgendwann einmal eine Ausbildung zu machen. Es ist gut, dass Sie sich diesen Traum bewahren können, bei all den Schwierigkeiten, mit denen Sie gerade zu kämpfen haben.»

Rom betont im Vergleich zu einem Therapieende in einer klassischen Psychoanalyse den Unterschied in der Gestaltung eines Therapieabschlusses mit Patienten, die eine Psychose durchgemacht haben. Besonders wichtig sei hier, dass man sich den Prozess der Therapie noch einmal in einer gemeinsamen Rückschau ansehe und mit möglichst grosser Offenheit darüber rede. Er empfiehlt, neue Themen nicht mehr anzuschneiden, sondern sich stattdessen dem Rückblick und Ausblick zu widmen (Rom 2007: 224). Bei der o.g. Patientin spielte der gemeinsame Rückblick eine wesentliche Rolle, da sie, wie viele andere schizo- 
phrene Patienten, begründete Schwierigkeiten hatte, die eigene Lebensgeschichte rückblickend auf einer kontinuierlich strukturierten Zeitachse gedanklich nachzuvollziehen. Hier hatte ich nun die Gelegenheit, sie beim Rückblick auf unsere gemeinsame Geschichte, die zumindest einen kleinen Ausschnitt ihrer eigenen Geschichte beinhaltete, zu unterstützen. Gemeinsam erinnerten wir uns an unsere erste Begegnung, die wir dualisierend betrachten konnten, indem ich ihr meine Erinnerung schilderte und auch ihr eigene Gedanken und Gefühle zu dieser Situation einfielen, die sie auch benennen konnte. Beispielsweise erinnerte ich mich, dass mir die Patientin bei unserer ersten Begegnung mitteilte, ich bräuchte mir nicht auch noch Sorgen um sie zu machen. Es würden sich schon genügend Menschen um sie sorgen. Sie selbst erinnerte sich zurück, dass sie vor unserem ersten Gespräch dachte, sie wolle nicht schon wieder zu einer Psychologin gehen. Ähnliche aber auch sich voneinander unterscheidende Wahrnehmungen unserer ersten Begegnung konnten als solche benannt werden und nebeneinander stehen bleiben. Auf die gleiche Weise gelang es uns im gemeinsamen Rückblick auf die Therapie, zwei verschiedene Therapiephasen voneinander zu unterscheiden: Eine erste Phase, in der wenig Kommunikation möglich war, in der das Misstrauen bei der Patientin noch stark ausgeprägt war, und eine zweite Phase, in der ein Dialog zwischen uns entstehen konnte, in dem die Patientin dank des neugewonnenen Vertrauens viele Themen einbringen konnte. Ihre positive Erfahrung mit mir im Standortgespräch konnten wir gemeinsam als Übergang von der ersten in die zweite Therapiephase benennen. Bedauerlicherweise mussten wir uns durch meinen Weggang aus der Institution, kaum, dass mit der Reduktion des Kontaktwiderstandes gute Grundlagen für eine fortschreitende Therapie gelegt worden waren, mit der anstehenden Abschiedsphase befassen. Dieses Bedauern drückte ich der Patientin gegenüber aus. Sie selbst wehrte das zunächst ab, indem sie sagte, sie könne ja mit ihrem Bezugsmitarbeiter das Gleiche reden, konnte aber ihre Trauer über das anstehende Therapieende auch benennen. Sie betonte, sie wolle dann, wenn ich nicht mehr da sei, keine neue Therapeutin bekommen. Gemeinsam erinnerten wir uns in der Rückschau, dass sie ursprünglich auch nicht zu mir in die Therapie kommen wollte, erst wenig Vertrauen hatte und später immer mehr. Diese Erfahrung, dass es ihr möglich ist, eine Therapeutin, der sie anfänglich nicht mit Sicherheit trauen konnte, als vertrauenswürdig zu erleben, nahm sie letztlich aus der Therapie mit. Sie konnte Vertrauen fassen, das auch mitteilen und daraufhin Themen in die Therapie einbringen, die sie beschäftigten. Im gemeinsamen Ausblick konnte sie u. a. die Erfahrung, dass der eigene Kontaktwiderstand im Therapieverlauf reduzierbar war, als eigene Fähigkeit des 
Vertrauen-gewinnen-Könnens begreifen, was ihr die Möglichkeit gibt, sich zu einem späteren Zeitpunkt in ihrem Leben wieder auf eine Therapie einzulassen.

\section{Literatur}

Rom, Josi (2007). Identitätsgrenzen des Ich. Einblicke in innere Welten schizophrenieund borderlinekranker Menschen. Göttingen: Vandenhoeck \& Ruprecht.

Benedetti, Gaetano (1975). Ausgewählte Aufsätze zur Schizophrenielehre. Göttingen: Vandenhoeck \& Ruprecht.

Benedetti, Gaetano (2003). Todeslandschaften der Seele. Göttingen: Vandenhoeck \& Ruprecht.

Möller, Hans-Jürgen; Laux, Gerd; Deister, Arno (2005). Psychiatrie und Psychotherapie. Stuttgart: Georg Thieme Verlag KG. 